\title{
A clinical minimum data set for primary dental care
}

\author{
R. S. Ireland, ${ }^{1}$ A. M. Jenner, ${ }^{2}$ M. J. Williams, ${ }^{3}$ and M. Tickle, ${ }^{4}$
}

\begin{abstract}
Objective To achieve consensus within primary dental care on the contents of a clinical minimum data set to measure oral health status.

Design Using the Delphi process a simple random sample of 30 LDCs and 10 CDS services in England were asked to rank a list of existing clinical indicators in order of their perceived importance as a means of measuring oral health. A nominated panel representing the stakeholder organisations of primary dental care reviewed this ranking and identified a core group of clinical indicators to be included in a clinical minimum data set.

Results An 80 percent response rate to the Delphi process was achieved. Consensus was reached on a core group of 10 indicators, which can provide information on patient's perceptions of pain, function and appearance, and professional measurements of caries, teeth present, periodontal disease, oral sepsis, presence of mucosal pathology and tooth wear.

Conclusions A representative sample of primary care dentists in England and the key representative organisations of primary dental care achieved consensus on the contents of a clinical minimum data set to record oral health status in primary dental care. This is a first step in standardising the measurement of oral health status across primary care.
\end{abstract}

Tformation management and technology (IM\&T) has an important role to play in the delivery of health care. ${ }^{1}$ This is particularly relevant with the emphasis currently being placed on audit and clinical governance in general dental practice. From a dental policy perspective, the need to understand the benefits which IM\&T can contribute to patient care was recognised when in 1996 the Department of Health commissioned the Interspec project, the aim of which was to develop a framework for IM\&T in dentistry. ${ }^{2}$ This project highlighted the need for consensus on a clinical minimum data set (CMDS) for the whole of primary dental care. A CMDS is a standardised, small number of variables (clinical indicators), which collectively attempt to summarise the oral health status of a patient. To be able to do this, a clear and agreed definition of oral health is needed. As long ago as 1947 the World Health Organization defined health as 'a state of complete physical, mental and social well-being and not merely the absence of disease or infirmity' ${ }^{3}$ Definitions of oral health now attempt to place an individ-

\footnotetext{
${ }^{1 *}$ Professor of Primary Dental Care, University of Liverpool, Liverpool ${ }^{2}$ Consultant in Dental Public Health, Manchester Health Authority, Manchester ${ }^{3}$ General Dental Practitioner, Manor Farm Dental Practice, Runcorn, North Cheshire ${ }^{4}$ Consultant/Senior Lecturer in Dental Public Health Manchester, Salford and Trafford Health Authorities, University of Manchester, Manchester ${ }^{*}$ Correspondence to: R. S. Ireland, Liverpool Dental Hospital and School of Dentistry, Pembroke Place, Liverpool, L3 5PS

E-mail:ireland@liverpool.ac.uk

REFEREED PAPER

Received 15.02.00; Accepted 22.08.00

(C) British Dental Journal 2001; 190: 663-667
}

ual's experience of their health in the context of their social environment. For example, the Oral Health Strategy for England defined oral health as 'a standard of health of the oral tissues which enables an individual to eat, speak and socialise without active disease, discomfort or embarrassment and which contribute to general well-being. ${ }^{4}$

A CMDS to measure oral health status will be a powerful tool to improve the quality of care delivered to patients. There have been very few initiatives directed at improving the quality of oral health care, although the Faculty of General Dental Practitioners made an important contribution in 1991 by producing a Self Assessment Manual and Standards (SAMS) publication for use in general dental practice. ${ }^{5}$ The interests of the dental profession have been mainly directed at improving the quality of dental interventions such as the quality of restorations or prostheses rather than measuring improvements in oral health. Quality represents a measure or degree of excellence and needs to be assessed against pre-defined criteria. It is therefore axiomatic that if quality is to be improved, indicators need to be defined against which any degree of improvement can be measured. It is equally important that clinicians have confidence in, and agree on the use of these indicators of oral health. ${ }^{6}$

An agreed primary dental care CMDS can be incorporated into computerised clinical systems, which will record the indicators automatically as part of the clinical patient record. This facility will allow practitioners, with little or no additional work, to retrospectively analyse changes in the oral health status of their patients. With the advent of clinical governance ${ }^{7}$, which has at its heart the improvement of the quality of the care provided to the patient, this capability will be essential ${ }^{7}$. In order to improve quality in primary dental care, clinicians must have an effective means of assessing the influence of any interventions they provide on the oral health status of their patients. A CMDS agreed by primary dental care clinicians would enable the clinical members of the dental team (dentists, hygienists and therapists) to do this. A standardised CMDS will not only facilitate self-audit but, as it is standardised across primary dental care, it will be possible for practitioners to compare the success of their interventions with those of their colleagues. Adopting this approach will support clinical decision making and foster an evidence-based perspective to the delivery of primary dental care. Preventive activity or treatment interventions that are shown to be ineffective could be modified or discontinued.

At a European level, research has been carried out to develop indicators of oral health status (a CMDS) for use in primary dental care. ${ }^{9}$ Whilst consensus has been achieved in Europe, these indicators have not been universally accepted in the UK and have so far not been adopted. This has been primarily due to the delay in integrating them into clinical software systems. However, work has continued on this development at a European level as a central part of the ORQUEST programme funded by the European Union. ${ }^{10}$ Although it is necessary to conform to European guidelines and strategies it is important to define a clinical data set appropriate to 
Table I Clinical indicators mean scores of the Ist and 2nd stages of the Delphi process, together with the final ranking produced from the 2nd stage.

\begin{tabular}{|c|c|c|c|}
\hline Indicator & $\begin{array}{l}\text { Ist stage } \\
\text { Mean }\end{array}$ & $\begin{array}{l}\text { 2nd stage } \\
\text { Mean }\end{array}$ & $\begin{array}{l}\text { Final } \\
\text { rank } \\
\text { orde }\end{array}$ \\
\hline Presence of dental pain & 7.5 & 7.5 & I \\
\hline Teeth with untreated caries & 7.4 & 7.4 & \\
\hline Sextants with deep pockets (6 mm or more) (BPE4) & 7.1 & 7.2 & 3 \\
\hline Teeth present per person & 7.1 & 7.1 & 4 \\
\hline Caries free persons & 6.8 & 7.1 & 5 \\
\hline Sextants with gingival bleeding (BPEI) & 6.8 & 7.0 & 6 \\
\hline Sextants with calculus (sub/supra) (BPE2) & 6.6 & 6.9 & 7 \\
\hline Sextants with shallow pockets (4-5 mm) (BPE3) & 6.7 & 6.9 & 8 \\
\hline Mobility of teeth (graded $0,1,2,3$ ) & 6.5 & 6.7 & 9 \\
\hline Teeth with suppuration & 6.4 & 6.5 & 10 \\
\hline Persons with pathological oral mucosal lesions & 6.4 & 6.4 & II \\
\hline Tooth surfaces with root caries & 6.3 & 6.3 & 12 \\
\hline Edentulous persons & 6.1 & 6.2 & 13 \\
\hline Teeth with fractures (dentine/pulp/root involvement) & 6.2 & 6.2 & 14 \\
\hline Persons with removable dentures (full/partial) & 6.0 & 6.0 & 15 \\
\hline $\mathrm{DMFT} / \mathrm{dmft}$ per person & 5.9 & 5.9 & 16 \\
\hline Root filled teeth (counted per tooth) & 5.9 & 5.9 & 17 \\
\hline DMFS/dmfs per person & 5.6 & 5.6 & 18 \\
\hline Teeth missing due to caries & 5.4 & 5.4 & 19 \\
\hline Teeth with loss of attachment (more than I/3 root) & 5.4 & 5.4 & 20 \\
\hline IOTN (Indication of Orthodontic Treatment Needs) & 5.4 & 5.4 & 21 \\
\hline Teeth with visible plaque & 5.3 & 5.3 & 22 \\
\hline \multicolumn{4}{|l|}{ Teeth with wear into dentine (abrasion/attrition) } \\
\hline per person & 5.3 & 5.3 & 23 \\
\hline Persons with malocclusions treated or referred & 5.2 & 5.2 & 24 \\
\hline Teeth with restorations having marginal defects & 5.0 & 5.0 & 25 \\
\hline Separate components of DMFT or dmfs & 5.1 & 4.9 & 26 \\
\hline 4 point pocket depth measurement per tooth & 4.9 & 4.9 & 27 \\
\hline Type of extracted teeth (permanent or deciduous) & 5.0 & 4.9 & 28 \\
\hline Filling overhang present & 4.8 & 4.8 & 29 \\
\hline Persons with 20 or more teeth & 4.8 & 4.7 & 30 \\
\hline$\%$ of tooth surfaces with secondary caries & 4.8 & 4.7 & 31 \\
\hline Tooth surfaces with visible plaque & 4.7 & 4.7 & 32 \\
\hline 6 point pocket depth measurement per tooth & 4.7 & 4.6 & 33 \\
\hline Pericronitis (per tooth) & 4.6 & 4.6 & 34 \\
\hline Teeth with gingival recession (in mm) & 4.3 & 4.3 & 35 \\
\hline Root fillings present (counted per root) & 3.9 & 3.8 & 36 \\
\hline Drifted tooth & 3.8 & 3.8 & 37 \\
\hline Open contact & 3.7 & 3.7 & 38 \\
\hline
\end{tabular}

the provision of both private and NHS primary dental care in the UK. A key concept to adhere to in developing a CMDS should be that the indicators must be clinically relevant rather than be dictated by technology. Equally, a CMDS cannot be imposed and clinicians must decide what information is important in order to measure the oral health of their patients. Therefore this study set out to achieve consensus on the contents of a clinical minimum data set of oral health status indicators across primary dental care in England.

\section{Method}

The first stage of the process was to undertake a comprehensive review of the clinical data sets used in the public and private sectors of primary dental care both in the European Union and in England. ${ }^{11,12}$ All UK dental software manufacturers were consulted with respect to the indicators recorded within their clinical systems. From this source material a list of 38 clinical oral health status indicators was defined.

The next stage of the process was to ask primary dental care clinicians to review the indicators and come to a consensus view of the relative clinical importance of the indicators. This was achieved using a Delphi process. ${ }^{13}$ Although some primary dental care is provided on a private contract basis, the majority of care is provided within the General Dental Services (GDS) and the Community Dental Services (CDS) and increasingly the Personal Dental Service (PDS). Local Dental Committees (LDCs) were considered most appropriate to represent the GDS/PDS input. The sampling frame was all LDCs in
England ( $\mathrm{N}=105)$, and a simple random sample of 30 LDCs was selected. Clinicians providing primary dental care in the CDS were represented by a simple random sample of 10 services, selected from all CDS services in England. The 3:1 proportion was chosen to reflect the relevant size of the two branches of the profession.

The list of clinical indicators was prepared in a questionnaire format and sent to the secretaries of all LDCs, and CDS Dental Service Managers (DSMs) selected, explaining the process and inviting their participation. All members of the selected LDCs and all CDS dentists of the selected community services were encouraged to participate in the scoring of the questionnaire collectively, using a set of guidelines. Prior to distribution the guidelines and questionnaire were piloted with two local LDCs (Liverpool and North Cheshire), and a local CDS service to identify any potential problems. Participants were asked to score each indicator according to a nine-point scale, ranging from:

- 1 = of no importance for inclusion in a CMDS, to

- $9=$ very important for inclusion in a CMDS.

calculating the mean score of each indicator assessed consensus across primary dental care. The rankings from the first round of the Delphi process were summarised by producing a national score and a score for each LDC/CDS and these were included in a repeat version of the questionnaire which was redistributed to the participants. During this second round of the Delphi process, the participants were invited to re-score each indicator by reference to the national score and ranking. Where there were outlying scores, the participants were asked to provide written justification for either modifying or not modifying their scores.

The final stage of the process was to quality assure the results of the Delphi process, agree a core group of indicators and provide guidelines for the clinical recording of the indicators. This was achieved using a nominal group methodology, the source material for which was the ranked list of indicators produced by the Delphi technique, plus the written responses provided by the Delphi participants.

For the nominal group meeting, representatives of organisations within the profession were asked to nominate and provide panellists for a one-day conference. In total 13 representatives joined the research team representing the British Dental Association, the Community Dental Service and the Faculty of General Dental Practitioners.

The nominal group process took the form of a full day meeting divided into two sessions. Prior to the date of the meeting the source material was provided for panellists to consider. The first session involved structured discussions in four groups on the ranking of the indicators to identify a core group of indicators. The findings from each group were reported back to the group as a whole and consensus was achieved for a core group of 10 indicators and a second group of additional indicators. The second part of the process was to establish recording guidelines for the indicators in clinical practice; this involved clarifying recording definitions for each indicator. Finally there was a discussion with regard to which professional organisation should take responsibility for periodically reviewing and updating the indicators.

The results from the nominal group meeting were fed back to the participants of the Delphi process for agreement and verification of the results and final comments.

\section{Results}

There was an $80 \%$ response from the LDCs $(\mathrm{N}=24)$ and a $100 \%$ response from the CDS $(\mathrm{N}=10)$ in the Delphi process. In order to maintain the 3:1 ratio of GDS to CDS, the CDS response was reduced to 8 by random selection. The GDS and CDS responses were aggregated for each indicator and a mean score calculated.

Table 1 contains the 38 clinical indicators derived from the review of existing data sets. Table 1 also displays the rankings of the 


\section{RESEARCH \\ information management}

Table 2 Consensus Clinical Individual Patient Oral Health Care Indicators: Core group and additional indicators.

\section{Core Group}

\begin{tabular}{ll} 
No. & Indicator \\
\hline 1 & Presence of oral pain \\
2 & Patient satisfaction with appearance \\
3 & Patient satisfaction with function \\
4 & Number of tooth surfaces with caries \\
5 & Number of teeth present per patient \\
6 & BPE Sextant Codes 0 - 4 \\
7 & Number of teeth with mobility > I mm \\
8 & Presence of infection (sepsis) of teeth and related structures \\
9 & Patients with oral mucosal lesions \\
10 & Teeth with wear into dentine per sextant per patient \\
\hline
\end{tabular}

\section{Additional Indicators}

\begin{tabular}{ll}
\hline II & Number of tooth surfaces with root caries \\
I2 & Caries free patients \\
I3 & Edentulous patients \\
14 & Teeth with fractures (dentine/pulp/root involvement) \\
15 & Patients with removable dentures (full/partial) \\
16 & DMFT/dmft per patient \\
17 & Root filled teeth (counted per tooth) \\
18 & DMFS/dmfs per patient \\
19 & Number of teeth missing due to caries \\
20 & No. of teeth with loss of attachment (more than I/3 root) \\
21 & IOTN (Indication of Orthodontic Treatment Needs) \\
22 & Number of teeth with visible plaque \\
23 & Patients with malocclusions treated or referred \\
24 & No. of teeth with restorations having marginal defects \\
25 & Separate components of DMFT or dmfs \\
26 & 4 point pocket depth measurement per tooth \\
27 & Type of extracted teeth (permanent or deciduous) \\
28 & Number of teeth with filling overhangs present \\
29 & Patients with 20 or more teeth \\
30 & \% of tooth surfaces with secondary caries \\
31 & Number of tooth surfaces with visible plaque \\
32 & 6 point pocket depth measurement per tooth \\
33 & Pericoronitis (per tooth) \\
34 & Number of teeth with gingival recession (in mm) \\
35 & Number of root fillings present (counted per root) \\
36 & Number of drifted teeth \\
37 & Number of open contacts \\
\hline
\end{tabular}

indicators from the first and second rounds of the Delphi process with the mean scores. Following the second stage of the Delphi process, 8 indicators moved up the ranking and 6 moved down. Table 2 shows the final core group of 10 indicators identified by the nominal group meeting, together with the 27 additional indicators. All of the discussion groups considered that two additional subjective indicators (patient's satisfaction with appearance and function) should be included in the core group of indicators. Although 'teeth with wear into dentine' was ranked 23rd by the Delphi process it was considered by the group to be of increasing importance with an ageing population and should therefore be included in the core group.

It was agreed that the definition of 'presence of dental pain' should be changed to read 'presence of oral pain' so as to include pain from oral structures other than the teeth. Presence of caries was considered to be an important indicator and 'tooth surfaces with caries' was substituted for 'caries free persons' to provide more detailed information on the caries status of the patient. The four indicators for Basic Periodontal Examination (BPE) were combined together to form one composite indicator

Of the 27 additional indicators, many were considered to be subsets of the core indicators. No indicators were excluded from the list defined by the LDC/CDS sample. It was accepted that primary care clinicians could record these additional indicators and further refine them into sub-sets of indicators which might be relevant to more specific groups of the patient database, eg children, and be linked with expert or specialist software systems.
The participants at the nominal group meeting defined recording guidelines for the core group of indicators which are listed in Table 3.

\section{Discussion}

The Delphi process is an inexpensive and rapid means of achieving consensus on any issue from large numbers of individuals or groups separated by distance. ${ }^{13}$ Therefore this methodology was ideal for gaining a representative view across primary dental care of the relative importance of the various clinical indicators present in existing data sets. Interestingly the only subjective indicator, presence of dental pain, appeared at the top of the ranking. This indicator was added to the list derived from the literature review by the research group following the suggestions of the dentists who participated in piloting the questionnaire prior to distribution. The dentists made a strong case for inclusion of pain, emphasising its importance as an indicator for treatment and the resolution of pain as an outcome indicator of effective care. This suggestion for inclusion was convincingly vindicated by the results of the Delphi process, which placed pain at the top of the ranking.

The list of clinical indicators drawn from existing data sets had a strong emphasis on the professional measurement of oral disease and conditions, and yet the nominal group, made up of the opinion formers and key representatives of professional organisations within primary dental care, added two further subjective indicators. The literature concerning the subjective measurement of oral health status has developed rapidly over the last decade. ${ }^{14,15,16}$ The fact that representatives of primary dental care insisted that subjective indicators of function and appearance should be included in the core group of indicators perhaps indicates that clinicians in England have recognised that the professional measurement of disease does not equate solely to the measurement of oral health. ${ }^{17,18}$ The importance of the subjective indicators for function and appearance included in the CMDS is suggested by their inclusion as indicators in the Denplan 'Excel' programme currently being piloted by approximately 660 general dental practitioners in England. ${ }^{18}$ These are very quick and simple to record compared with many of the subjective indices proposed in the literature. ${ }^{14,15,16}$ It must be remembered that the utility of the indicators included in the CMDS depends on whether they can be captured without impinging on the efficient running of a busy dental practice, and also if the outputs of the indicators are meaningful to clinicians.

There could be a criticism of the role of the nominal group in this project, given the seemingly greater credence granted to the views of this group in comparison with the results received from a representative sample of primary dental care clinicians across England. For example, the nominal group added two indicators and moved the indicator relating to tooth wear, which was ranked 23 rd in the Delphi process, into the core group of indicators. In defence of these apparently arbitrary decisions, the role of the nominal group was to quality assure the results of the Delphi process and to decide on a core group of indicators. Whilst the Delphi process is an excellent means of reaching a consensus quickly, it provided limited feedback from primary dental care providers simply because the questionnaire used was made up of closed questions. A structured discussion permits a much more in depth investigation of an issue. Also the nominal group was made up of representatives of the key organisations representing primary dental care in England and for the CMDS to be accepted and used, it is important for these organisations to support the results of this research.

The final list of 10 core indicators making up a CMDS can be summarised as capturing information relating to six distinct areas:

- Patient perceptions of their oral health status

- Clinical recording of caries

- Clinical recording of periodontal disease

- Clinical recording of oral sepsis

- Clinical recording of oral pathology

- Clinical recording of tooth wear 
Table 3 Recording guidelines for a Core Group Clinical Oral Health Care Indicators to make up a CMDS for primary dental care.

\begin{tabular}{|c|c|c|c|c|}
\hline Indicator & Recording definition & Field Type & Field length & Format \\
\hline Presence of oral pain & Is your mouth free from pain? & Character & I & $\mathrm{Y} / \mathrm{N}$ \\
\hline Patient satisfaction with appearance & Are you happy with the appearance of your teeth? & Character & I & $\mathrm{Y} / \mathrm{N}$ \\
\hline Patient satisfaction with function & Can you comfortably chew an unrestricted diet? & Character & I & $\mathrm{Y} / \mathrm{N}$ \\
\hline Number of tooth surfaces with caries & $\begin{array}{l}\text { A tooth is considered to be carious if in the opinion of the examiner, after } \\
\text { visual or other examination methods, there is clinical caries present with } \\
\text { enamel breakdown. Caries is recorded as present when a lesion in a pit } \\
\text { or fissure, or on a smooth surface, has a detectably softened floor, } \\
\text { undermined enamel or softened wall. Non-carious restored teeth } \\
\text { and teeth extracted due to caries should be excluded. The following } \\
\text { conditions should be charted as sound: } \\
\text { White or chalky spots. } \\
\text { Discoloured or rough spots (but not soft to touching with the probe). } \\
\text { Stained pits or fissures in the enamel. } \\
\text { Dark, shiny, hard, pitted areas of enamel. } \\
\text { Lesions due to abrasion }\end{array}$ & Numeric & 3 & \\
\hline Number of teeth present per patient & $\begin{array}{l}\text { A tooth should be considered as present when any part of it is visible } \\
\text { or can be touched with the tip of a probe without unduly displacing soft } \\
\text { tissue }\end{array}$ & Numeric & 2 & \\
\hline BPE sextant codes & $\begin{array}{l}\text { Code } 0 \\
\text { No bleeding on probing is demonstrated by introducing a CPITN probe } \\
\text { into the gingival sulcus and moving it gently around the circumference of } \\
\text { the tooth using no more than } 25 \mathrm{mg} \text { pressure (about that which causes } \\
\text { discomfort when a probe is introduced below the cuticle of the nail bed). } \\
\text { No calculus is detected } \\
\text { Code I } \\
\text { Bleeding on probing. A site is scored as positive if bleeding ensues within } \\
30 \text { secs. No calculus is detected. } \\
\text { Code } 2 \\
\text { Where sub or supra-gingival calculus is detected with a CPITN probe. } \\
\text { Black band is completely visible. } \\
\text { Code } 3 \\
\text { Black band of CPITN probe partially but not completely obscured. } \\
\text { Code } 4 \\
\text { Black band of CPITN probe is completely obscured. }\end{array}$ & Numeric & $\mathrm{I}$ & $0,1,2,3,4$ \\
\hline Number of Teeth with mobility $>$ I mm & $\begin{array}{l}\text { I mm buccal/lingual movement in response to pressure from hand } \\
\text { instrument on either side of tooth. } \\
\text { Presence of infection (sepsis) of teeth and related structures } \\
\text { a discharging/non-discharging sinus. Pus expressed from a periodontal } \\
\text { pocket on digital pressure or probing. Recorded per patient. }\end{array}$ & $\begin{array}{r}\text { Numeric } \\
\text { Presence of } \\
\text { Character }\end{array}$ & 2 & $(\mathrm{Y} / \mathrm{N})$ \\
\hline Presence of oral mucosal lesions & Any visually detected lesion of the oral mucosa. Recorded per patient. & Character & I & $(\mathrm{Y} / \mathrm{N})$ \\
\hline $\begin{array}{l}\text { Teeth with wear into dentine per } \\
\text { sextant per patient }\end{array}$ & $\begin{array}{l}\text { Where more than one third of the enamel has been lost on either the } \\
\text { buccal, lingual or occlusal surfaces or where enamel has been lost with } \\
\text { dentine exposed incisally or where there is a cervical defect of more } \\
\text { than } 2 \mathrm{~mm} \text {. }\end{array}$ & Character & I & $(\mathrm{Y} / \mathrm{N})$ \\
\hline
\end{tabular}

The professionally measured indicators contained in the CMDS did not include an orthodontic indicator. The Indication of Orthodontic Treatment Need (IOTN) index was ranked at 21st position in the Delphi process. In the nominal group discussions the view taken was that the specialist subject of orthodontics would require a separate CMDS and work has been progressing on this at a European level. ${ }^{19}$

The initial list included indicators at various levels of assessment, namely:

- Tooth surface

- Tooth

- Sextant

- Individual patient

- Population (aggregates of patients).

It is envisaged that the CMDS embedded within a dental software system could be disaggregated or compounded, according to the requirements of the clinician. Similarly ordinal measures of severity could be recorded for the subjective indicators and categorical subdivisions could be included, for example for the 'presence of oral mucosal lesions' indicator.

Standardised measurement of oral health status also has the potential to support strategic planning by providing regular infor- mation on the health needs of the local population. ${ }^{8}$ The need for a common currency of information across primary dental care has become even more evident with the development of Personal Dental Services (PDS) and Dental Access Centres (DACs) working under contract with Health Authorities to help deliver oral health improvement targets included in health improvement programmes. Using a standardised CMDS, clinicians could compare their performance against quality or health improvement goals. These goals could be set at individual practice or clinic, Primary Care Trust or national levels.

In conclusion a representative sample of primary care dentists in England, together with the key representative organisations of primary dental care achieved consensus on a clinical minimum data set to measure oral health status in primary dental care. However to be of value to clinicians and to help improve the care provided to patients the indicators need to be used and evaluated in routine clinical practice. The list of core indicators will inform the Faculty of General Dental Practitioners expert working body currently preparing guidelines on clinical record keeping. At the nominal group meeting it was agreed that the Faculty of General Dental Practitioners would be responsible for reviewing the indicators every three years and updating them where appropriate with input from all representatives of primary dental care, eg the BDA, FGDP, and the CDS. 


\section{information management}

This study was funded by the NHS National Primary Dental Care ReD Programme. The authors wish to thank David Jones and Ian Soady from the NHS Information Authority and Tom Hart from the Dental System Suppliers Association for their support and expertise. They would also like to thank the LDCs and CDS services, the British Dental Association and Faculty of General Dental Practitioners of the Royal College of Surgeons of England for their participation in the study.

1 Department of Health Information for Health - An information strategy for the modern NHS, 1998 HMSO London.

2 Tickle M, Jenner A M, Rolfe P, Kay E J. Towards a standardised information system for dentistry Br Dent J 1998; 184: 472-473

3 World Health Organization. Alma-Ata Declaration 1947

4 Department of Health The Oral Health Strategy for England 1994 HMSO London

5 Royal College of Surgeons, England. Self Assessment Manual and Standards. Clinical standards in general dental practice. Faculty of General Dental Practitioners. London. 1991.

6 Gift H C. Oral health outcomes research - challenge and opportunities. Chapter 3. G Slade Ed. Measuring oral health and quality of life. Chapel Hill: University of North Carolina, Dental Ecology 1997.

7 HMSO. A First Class Service; Quality in the new NHS. 1998. London

8 Tickle M, Jenner A M, Kay E J. A model to meet the strategic information needs of dentistry in England Comm Dent Health 1997; 14: 17-18
9 Oratel-Telematic System for Quality Assurance in Oral Health Care. Implementation of the ORATEL Software Tools. CEC Project A-2029 1994.

10 ORQUEST. A telematic System for Oral Health Quality Enhancement. HC 1037, Telematics Applications Programme. HC 1037

11 Oratel-Telematic System for Quality Assurance in Oral Health Care. Project report and final demonstrators. CEC Project A-2029 1994.

12 Ireland R S. Clinical quality assurance indicators for oral health status and treatment of a group of older adults. Br Dent J 1998;185:192-195

13 Pill J. The Delphi Method: substance context, a critique and an annotated bibliography. Socio-Economic Planning Science 1975; 5: 57-71.

14 Atchison K, Dolan T. Development of the Geriatric Oral Health Assessment Index. J Dent Education, 1990; 54: 680-687.

15 Locker D, Miller Y. Evaluation of subjective oral health status indicators. J Public Health Dent, 1994; 54: 167-76.

16 Slade G D, Spencer A J. Development and evaluation of the Oral Health Impact Profile. Comm Dent Health, 1994; 11: 3-11.

17 World Health Organisation The Constitution of the World Health Organisation. WHO Chron. 1947;1, 29. Geneva, World Health Organisation

18 Denplan Excel Programme 1999. Denplan Ltd. Denplan Court, Winchester.

19 Njio B J, Stenvik A, Ireland R S, Prahl-Andersen B. EURO-QUAL European Orthodontic Quality Manual. IOS Press. 